Conf $890426--9$

Rereived by OSTh-BL-2098:

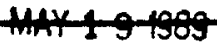

L Lawrence Berkeley Laboratory

UNIVERSITY OF CALIFÓRNIA

Physics Division

Presented at the Matcrials Research Society Conference,

Sin Diego, CA, April 24-28, 1989, and to be published

in the Proceedings

'The Application of Thick Hydrogenated Amorphous Silicon Layers to Charged Particle and X-Ray Detection

V. Perez-Mendez, G. Cho, I. Fujieda, S.N. Kaplan, S. Qureshi, and R.A. Street

April 1989

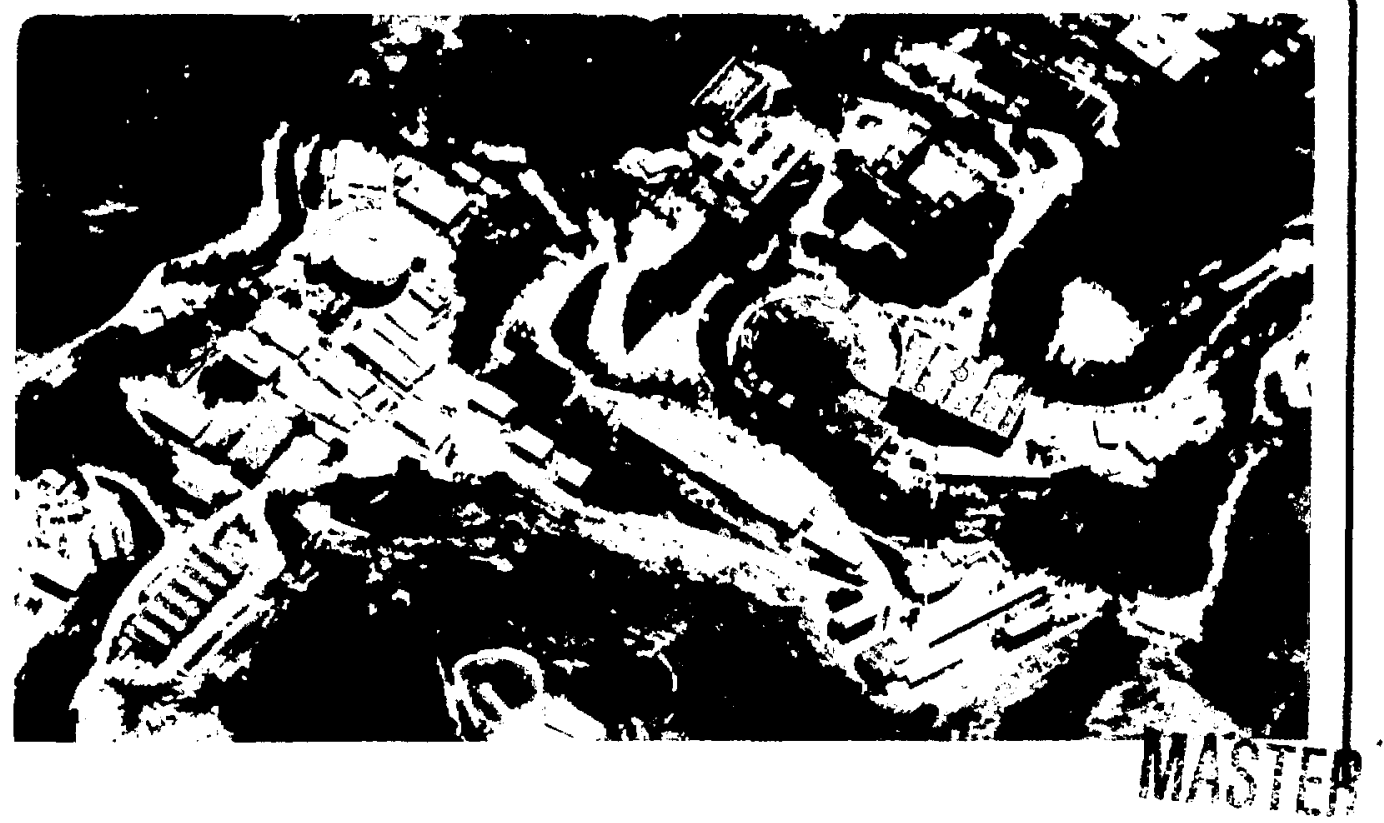

Prepared for the U.S. Department of Energy under Contract Number DE-AC03-76SF00098. 
LBL- -26998

DE89 011777

\title{
THE APPLICATION OF THICK HYDROGENATED AMORPHOUS SILICON LAYERS TO CHARGED PARTICLE AND X-RAY DETECTION*
}

April 10, 1989

V. PEREZ-MENDEZ, G. CHO, I. FUJIEDA, S.N. KAPLAN, S. QURESHI, Lawrence Berkeley Laboratory, University of California, Berkeley, California 94720

R.A. STREeT, Xerox P.A.R.C., Palo Alto, California 94304

\section{DSCLAIMIR}

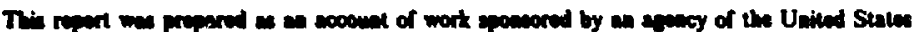

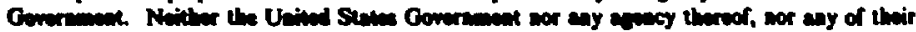

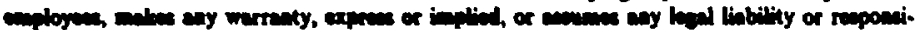

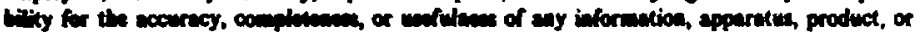

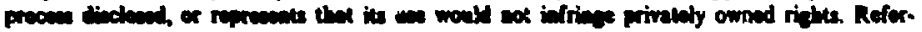

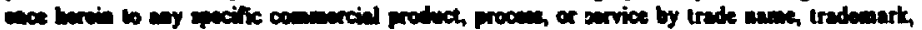

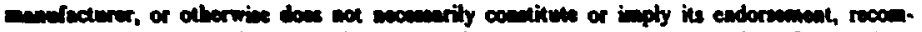

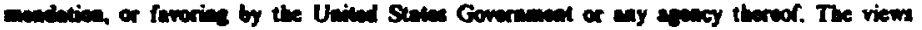

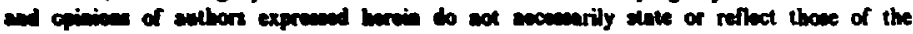

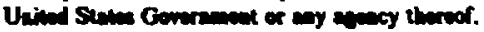

*This work was supported by the Director, Office of Energy Research, Office of High Energy and Nuclear Physics, Division of High Energy Physics of the U.S. Department of Energy under contract \#DE-AC03-76SF00098. 


\section{ABSTRACT}

We outline the characteristics of thick hydrogdenated amorphous silicon layers which are optimized for the detection of charged particles, $x$-rays and $y$-rays. Signal amplitude as a function of the linear energy transfer of various particles are given. Noise sources generated by the detector material and by the thin film electronics - a-Si:H or polysilicon proposed for pixel position sensitive detectors readout are described, and their relative amplitudes are calculated. Temperature and neutron radiation effects on leakage currents and the correspor:ting nise changes are presented.

\section{INTRODUCTION}

Thin layers of hydrogenated amorphous silicon (a-Si:H) of thickness $-1 \mu \mathrm{m}$ have found extensive application in solar cells and in thin film transistors (T.F.T.). A well known application of thick $-40 \mu \mathrm{m}$ layers of a-Si:H is to electrophotography devices. In these devices the usual configuration is a $p-i-n$ diode with thin $p$ and $n$ doped layers, and the bulk consisting of intrinsic a$\mathrm{Si}: \mathrm{H}$. We have studied the possibility of using a-Si:H reverse biased diode arre /s for charged particle, $x$-ray and $\gamma$-rny detectors. These would find use in particle physics research, in biological, and in medical imaging applications.

In particle physics a common requirement is to locate the trajectory of relativistic and slower particles by determining their passage through a number of layers of position sensitive detectors. These detector layers have consisted in the past of gas filled multiwire proportional chambers, scintillation counters and of crysal silicon diode arrays. The potential usefulness of a-Si:H detectors lies in the ease of maling large area devices by PECVD techniques, defining the shape and size of the detector element by lithography, and by being able to couple thin film readout electronics - a-Si:H or polysilicon directly to the detector "pixels" or "strips" as shown schematically in Fig. I. In the following sections we describe the results of our measurements using alpha particles, low energy $1-2 \mathrm{MeV}$ protons, $1 \mathrm{MeV}$ minimum ionizing electrons, $x$-rays and $\gamma$-rays.
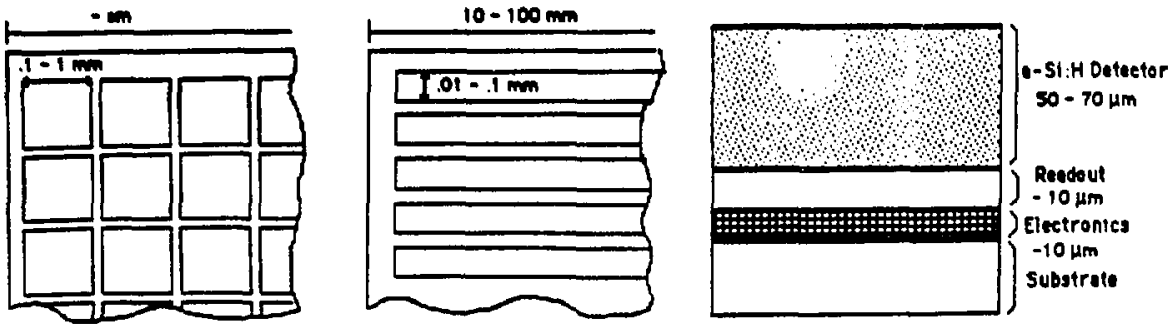

Fig. 1 Schematic coupling of a-Si:H detector pixels or strips to thin film electronics. Each metal pad is coupled to an individual charge sensitive amplifier below. 


\section{DETECTION OF CHARGED PARTICLES}

For the detection of charged particles traversing a sensitive layer of a-Si:H suitably doped to form a $p$-i-n diode we have the following requirements:

a. The sensitive layer has to be thick enough to ensure that the charged particle produces a sufficient number of electron-hole pairs that will result in a signal appreciably larger than noise. For the detection of minimum ionizing particles, which are of interest in high energy particle physics, we have shown that layers $50-70 \mu \mathrm{m}$ thick could be sufficient for this purpose. This conclusion stems from our measurement (given below) of the average energy $w=6.0 \pm 0.2 \mathrm{eV}$ required to produce l e-h pair in a-Si:H, which results in a yield of $60 \mathrm{e}-\mathrm{h}$ pairs/4m of detector layer.

b. The $i$ layer of the thick $p-i-n$ diodes has to be fully depleted in order to collect the bulk of the $e-h$ pairs produced by the transit of a charged particle. This implies that the $E$ field should be $\geq$ $10^{2} \mathrm{~V} / \mathrm{cm}$ throughout the layer in order to ensure that a large fraction of both electrons and holes are collected. The electric freld drops off as a function of distance due to the residual (+ve) charges left in the ionized dangling bond states of the $i$ layer. Figure 2 shows the electric field versus distance for various bias voluges for an assumed value of ionized dangling bond states $N^{*}=7 \mathrm{x}$ $10^{14} / \mathrm{cm}^{3}$ [1]. Recent messurements - presented at this meeting [2], show that only a small fraction $-30-35 \%$ of the dangling bonds as measured by E.S.R. are ionized. Hence with the present quality of material evailable to us from Xerox PARC (Palo Alto, CA), Glasstech-Solar (Wheatridge, $\mathrm{CO}$ ), Plasm Physics (Locust Valley, NY) and others, with dangling bond densities of $1-2 \times 10^{13} / \mathrm{cm}^{3}$ we are assured of full depletion for our proposed thick detectors for elementary particle physics experiments.

We confirm these projections by our measurements on p-i-n diodes $38 \mu \mathrm{m}$ thick. Figure 3 shows the signal produced by light pulses of $\lambda=665 \mathrm{~nm}$, whose mean free path for absorption in a-Si:H is $\sim 1 \mu \mathrm{m}$, and from $880 \mathrm{~nm}$ light with an absorption mean free path $>100 \mu \mathrm{m}$. The signal collection threshold from the $665 \mathrm{~nm}$ light incident on the $\mathrm{n}$ surface shows the minimum bias needed for the electric field to extend across the layer thickness. This allows us to calculate the density of ionized dangling bonds and to confirm the onset of full depletion[3]. The $880 \mathrm{~nm}$ light simulates the $\mathrm{e}, \mathrm{h}$ production, by the transit of a minimum ionizing particle.

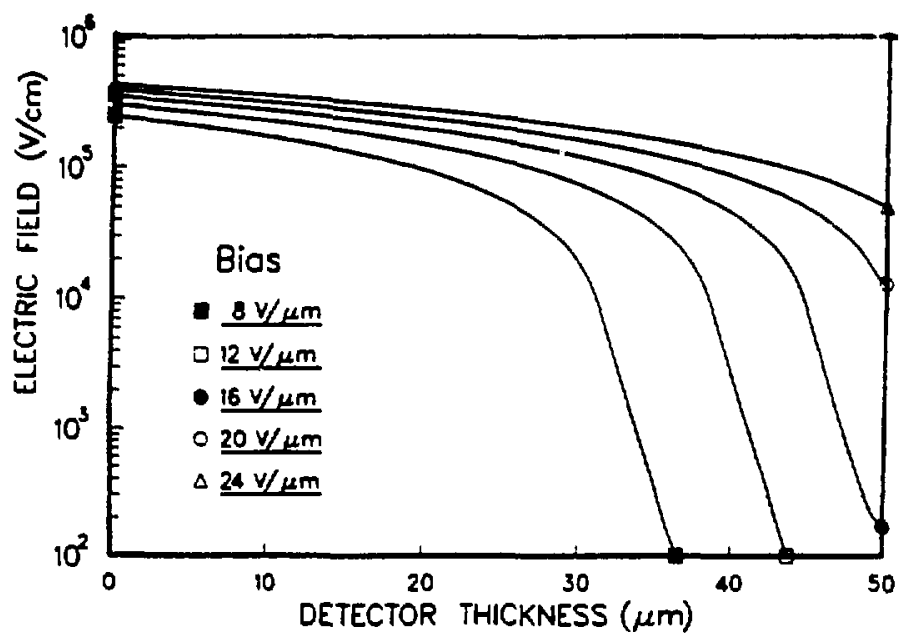

Fig. 2 Electric field shape in $50 \mu \mathrm{m}$ thick $\mathrm{i}$ layer of $\mathrm{p}-\mathrm{i}-\mathrm{n}$ diode for various bias voltages. 
c. The detector material itself generates noise, primarily due to the fluctuations in the capture and release of the e,h traversing the detector material. Figure 4 shows the noise and reverse bias current produced in a $38 \mu \mathrm{m}$ p-i-n layer. As is conventional in particle physics both signal and noise are recorded by charge sensitive amplifiers followed by a shaping network; in this case we used $3 \mu$ sec $R C-(C R)^{3}$ shaping.

Noise calculations indicate that the rapid increase of the noise amplitude above some bias level as seen in Fig. 4 is not the shot noise from the leakage current but is probably due to some incipient breakdown mechanism associated with defects in the sample layer. Microscope pictures (Fig. 5) taken of the surface of a typical metal coated $p$-i-n diode show bubbles and other aberrations which could explain this partial breakdown effect.

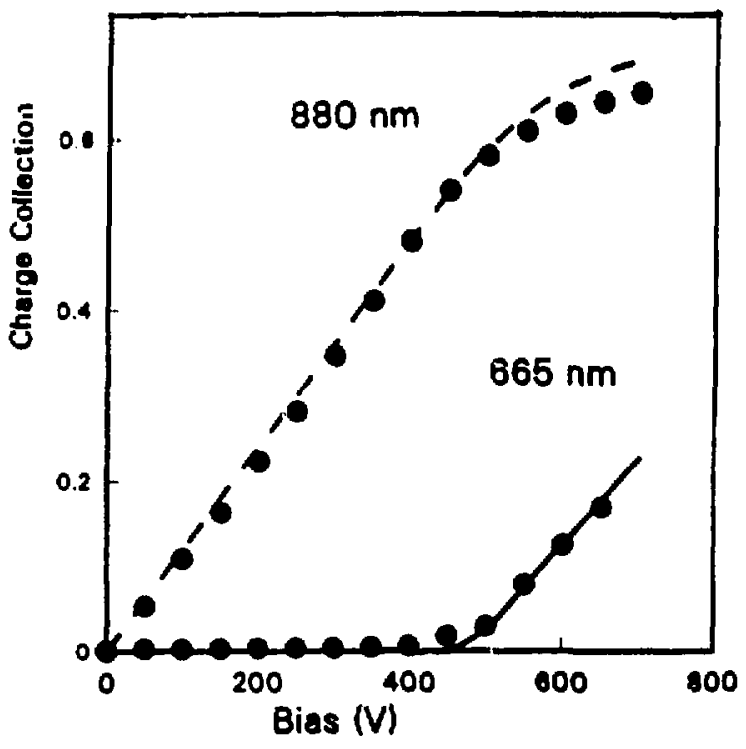

Fig. 3 Signal output versus bias for laser light incident on $\mathrm{p} \cdot \mathrm{i}-\mathrm{n}$ diode solid, dashed lines calculared using $\mu_{e}=1.2 ; \mu_{h}=0.004$ $\mathrm{cm}^{2} / \mathrm{Vs} \cdot(\mu \tau)_{\mathrm{e}}=1.0 \mathrm{x}$ $10^{-7} ; \mu \tau_{h}=1.2 \times 10^{-8}$ shaping time $=3 \mu \mathrm{sec}$.

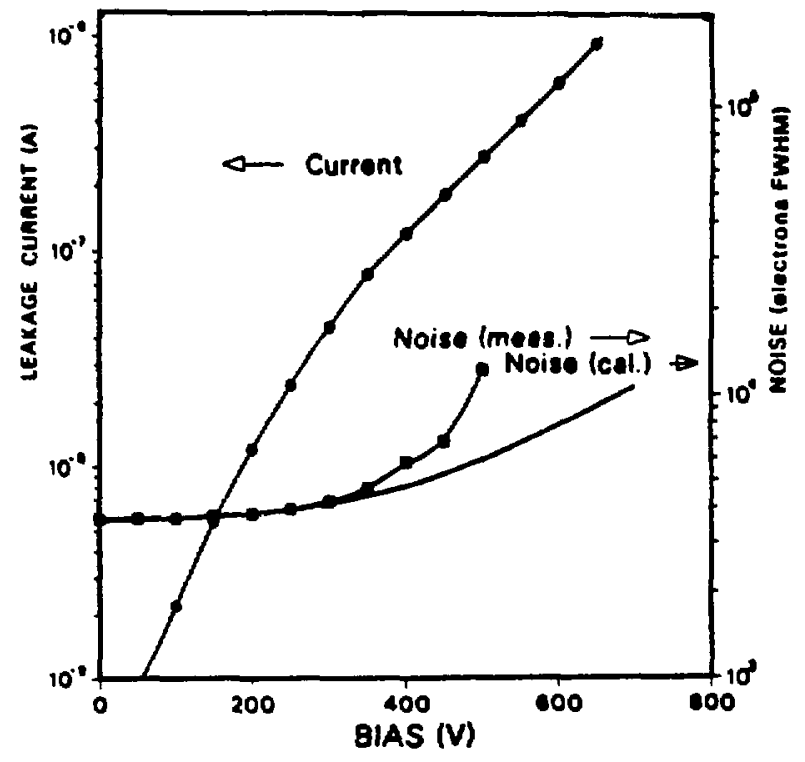

Fig. 4 Reverse current and noise of a $38 \mu \mathrm{m}$ thick $\mathrm{p}$-i-n detector diode. The initial increase of noise follows the calculated shot noise due to the current. The extra noise at higher biases may be due to incipient breakdown. 


\section{MEASUREMENTS WTTH ALPHA PARTICLES, LOW ENERGY PROTONS AND MINIMUM IONIZING ELECTRONS.}

We started this research using relatively thin p-i-n diodes and exposed them to 5-6 MeV alpha particles from Americium sources. We found. [4] and other groups also confirmed this[5] that the alpha particle signals were quite small relative to what would be expected from such a high eneryy deposition. As shown in Fig. 6 the observed signals were due to collection of 20,000-30,000 electrons. This effect is probably due to the large recombination rate of $e, h$ in the highly dense ionization channel produced by the alpha tracks. Such charge recombination is also seen in xtal Si detectors when exposed to very highly ionizing particles such as tission fragments. The signals produced by $l$ and $2 \mathrm{MeV}$ protons with linear energy (LET) transfers of 46 and $28 \mathrm{KeV} / \mathrm{micron}$ respectively show less signal loss due to recombination effects, than the alpha particle. Minimum ionizing ( -1 MeV electrons produce signal levels (Fig. 6) consistent with $W=6 \mathrm{eV}$. The signials produced by $\sim 1 \mathrm{MeV}$ electrons in 12.29 and $38 \mu \mathrm{m}$ thick detectors are shown in Fig. 7. The fix:t that the signal amplitude is proporional to the detector thickness and is consistent with our measured value of $w$ indicates that there is litte or no charge recombination. These measurements were done by summing signals and noise over a number of pulses [6] in order to overcome the large noise threshold of the charge sensitive amplifiers that we used.
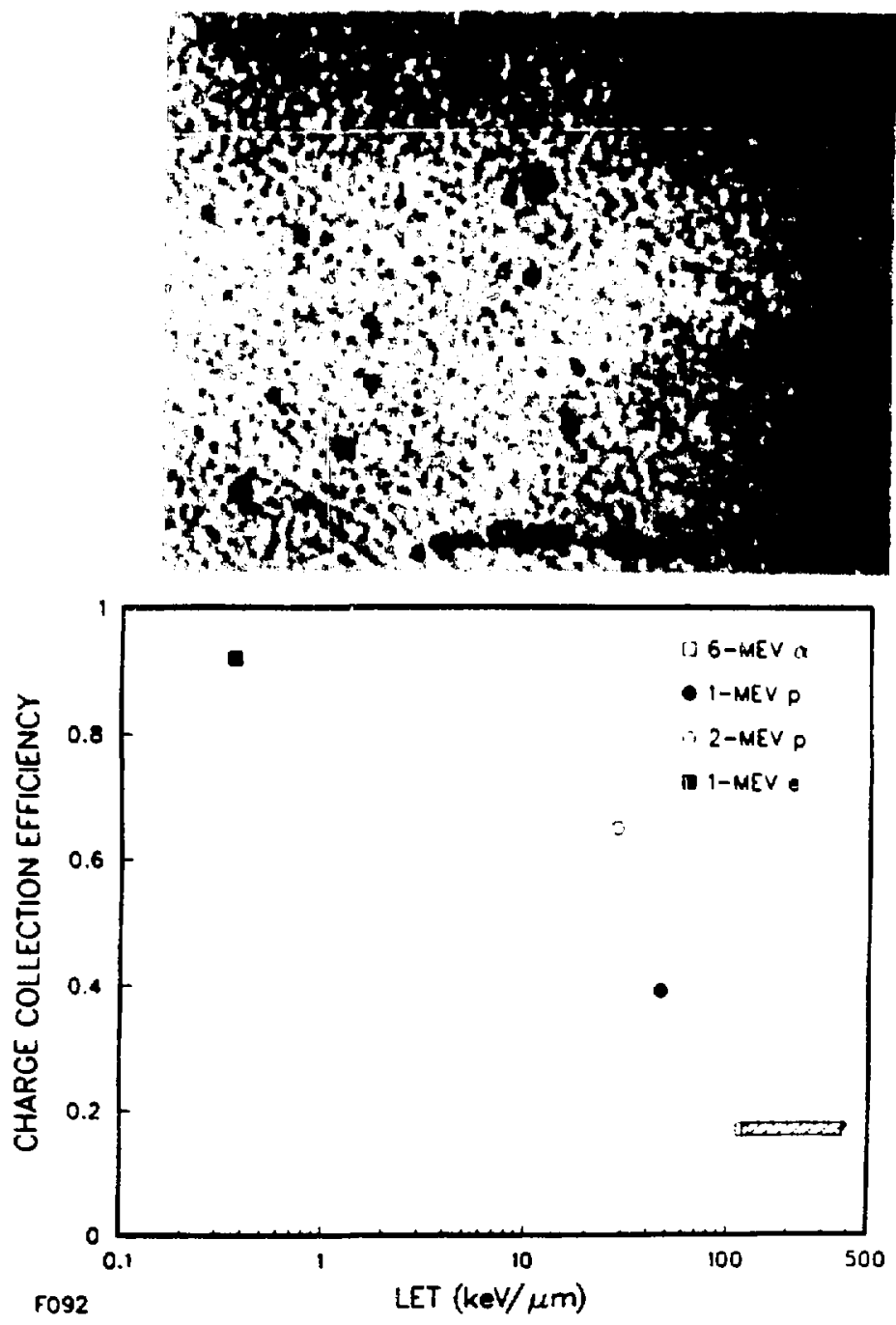

Fig. 5 Photograph of $38 \mu \mathrm{m} p-i \cdot n$ diode surface showing bubbles or dust imperfections.
Fig. 6 Signal levels produced by alpha particles, 1, 2, MeV protons and by minimum ionizing 1 MeV electrons. Expected signal calculated from $W=6$ $e V$ to produce $e, h$ pair. Charge recombination seen for alphas and low energy proton. 


\section{DETECTION OF X-RAYS IN a-Si:H}

The signals produced by $x$-ray pulses were detected in the set up shown in Fig. 8 . One to three $\mu$ sec long pulses of $x$-rays produced in a $20 \mathrm{kv}$ molybdenum anode $x$-ray tube were incident on 5 and $10 \mu \mathrm{m} n-\mathrm{i}-\mathrm{p}$ a-Si:H diodes, as well as on $180 \mu \mathrm{m}$ thick xtal silicon detectors. By comparing the signal amplitudes and energy deposited in the a-Si:H and xtal silicon and using the known value of $w\left(x t a l S_{i}\right)=3.62 \mathrm{eV}$, we obtain the result $w(a-S i: H)=6.0 \pm 0.2 \mathrm{eV}$ pairs. [7] The value of $w$ depends on the width of the band gap of the semiconductor diode and is consistent with the interpolated value obtained from measurements on various semiconductors [8].

We, and others [9] have also detected signals produced by $130 \mathrm{KeV} \gamma$-rays on the thicker aSi:H detectors where the $\gamma$-rays eject electrons of a few tens of KeV energy by the Compton eftect.

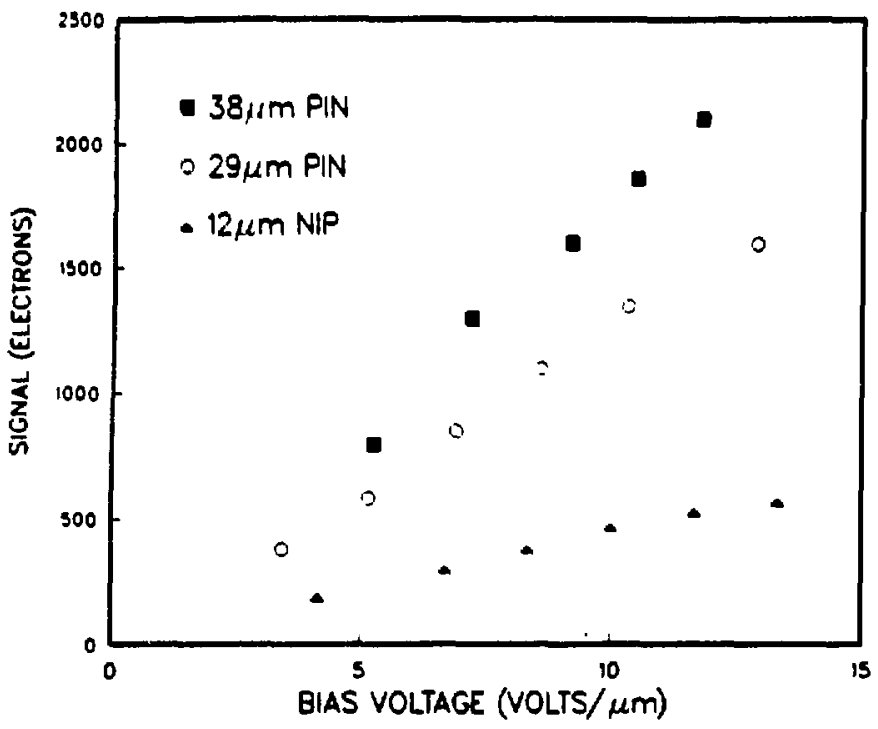

Fig. 7 Signals produced by minimum ionization electrons $\sim \mathrm{l} \mathrm{MeV}$ as function of bias voltage on $12,19,38 \mu \mathrm{m}$ thick $\mathrm{p}-\mathrm{i}-\mathrm{n}$ diodes.

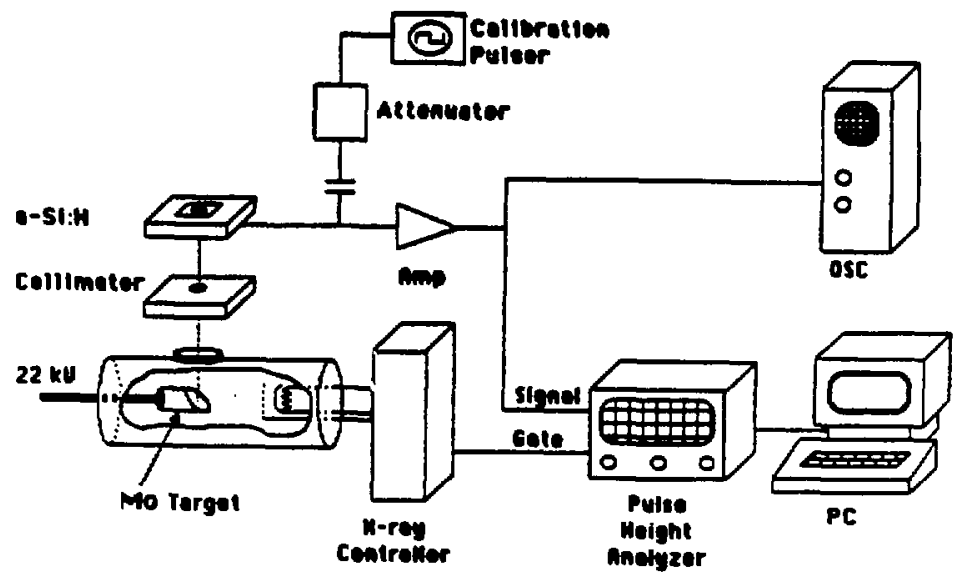

Fig. 8 Schematic of pulsed $x$-ray set up used for measuring $W$ in a-Si:H p-i-n diodes. 
A more sensitive method for detecting $x$-ray fluences, single high energy $\left(E_{\gamma} .100 \mathrm{KeV}\right.$ ) $\gamma$-rays and individual minimum ionizing charged part: $s$ is shown in Fig. 9. A $\mathrm{C}_{\mathbf{S}}$ I [Thallium or Sodium dopedl scintillation layer, deposited on top of the a-Si: $\mathrm{H} \mathrm{p}-\mathrm{i}-\mathrm{n}$ layer converts the energy of the incident radiation into light in the visible range which in turn produces e-h pairs in the a-Si:H with effective detection efficiency $>70 \%$ for $350 \mathrm{~nm}<\lambda<750 \mathrm{~nm}$. [10] The light emission efficiency of doped $C_{5} I$ ranges from $30,000-50,000$ visible light photons per MeV of deposited energy [11], with decay times between $0.6-1.1 \mu \mathrm{sec}$. For high spatial resolution detection with pixel sizes $\leq$ $50 \mu \mathrm{m}$, the sideways spread of the light can be prevented by suitable heat treatment of the evaporated $C_{s} I$ layers which produces columnar structures in the phosphor.[12] For alpha particle detection, layers $15-20 \mu \mathrm{m}$ are sufficient. For $x$-rays, $\gamma$-rays and minimum ionizing particles, layers $300-400 \mu \mathrm{m}$ thick would be adequate for the detection of single particles.

\section{NOISE DEPENDENCE ON ENVIRONMENT: TEMPERATURE AND RADIATION EFFECTS}

For single particle detection it is desirable to maintain a signal/noise ratio $\geq 10$. Since the signal is fixed by the detector thickness it is necessary to keep the noise at a sufficiently low level. In a physics detector the ambient temperature could rise due to power dissipation in the readout electronics for the pixel arrays. Figure 10 shows the reverse current and noise increase as a function of temperature. Since the overall noise is the sum in quadrature of the Nyquist, shot.and flicker noise, the shot noise produced by the thermal increase in reverse current becomes a major contribution at higher temperatures. The appropriate threshold in noise increase around $60^{\circ} \mathrm{C}$ is satisfactory for most applications, and can be increased by shortening the shaping time and or decreasing the reverse current by enhancing the $p$ layer barrier which can be done by adding carbon [13].
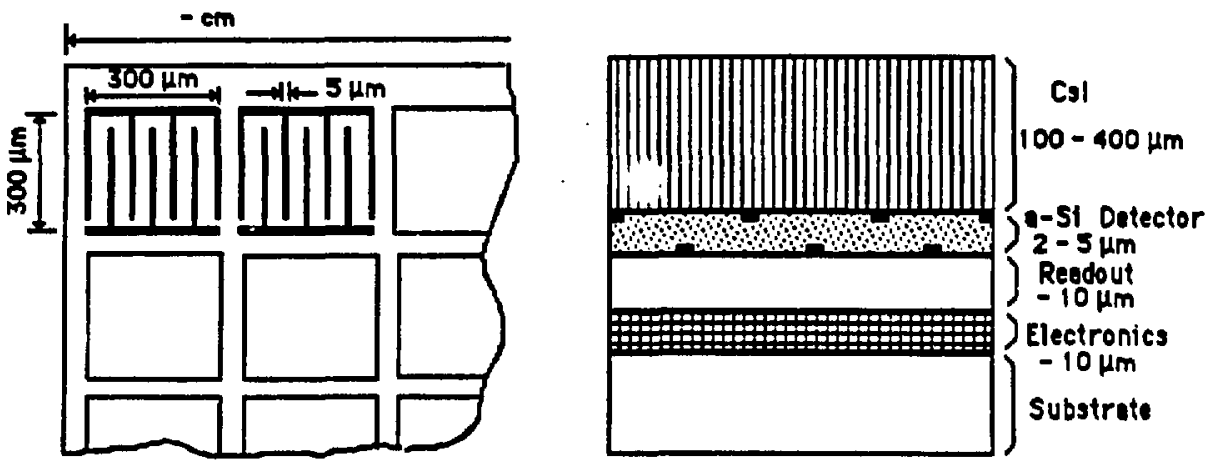

Fig. 9 Projected pixel device using $C_{s} l$ evaporated layer as detector. The $C_{s} I$ layer converts the incident radiation to visible light which is detected by thin $\mathrm{a}-\mathrm{Si}: \mathrm{H}$ diodes with interdigitated electrodes to reduce capaciance.

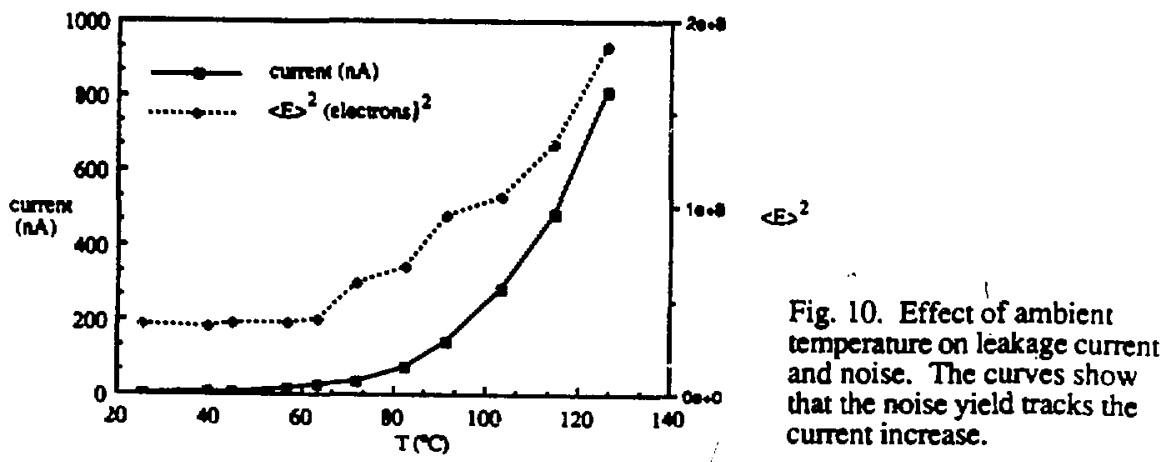


In Fig. 11 we show the increase in leakage current and in shot noise produced by radiation damage induced by $\sim 1 \mathrm{MeV}$ neutrons.[7] The noise and leakage current increases were minimal up to the largest fluxes $\sim 5 \times 10^{14}$ neutrons $/ \mathrm{cm}^{2}$, that we used. These increases are almost completely annealable by heating the samples at $-200^{\circ} \mathrm{C}$ for 2 hours. Data using proton exposures up to $10^{15}$ protons $/ \mathrm{cm}^{2}$ show similar results [14].

The relative insensitivity of a-Si:H detectors to these environmental effects as compared to xtal Si detectors enhances their usefulness for large area pixel arrays in particle physics research.

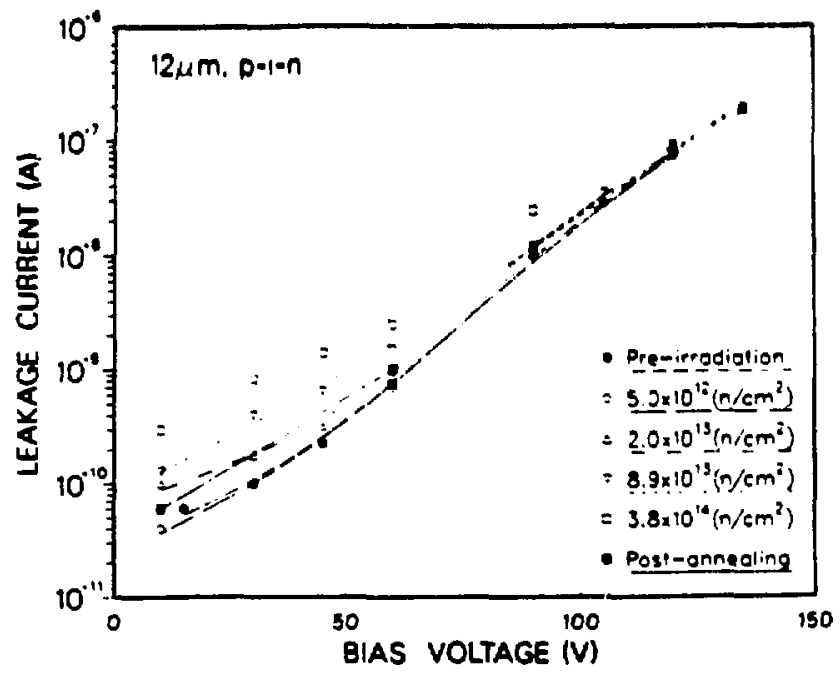

Fig. 11 Radiation damage induced by $\sim 1$ MeV neutrons and subsequent annealing (a) leakage current increase (b) change alpha particle signal and noise increase.

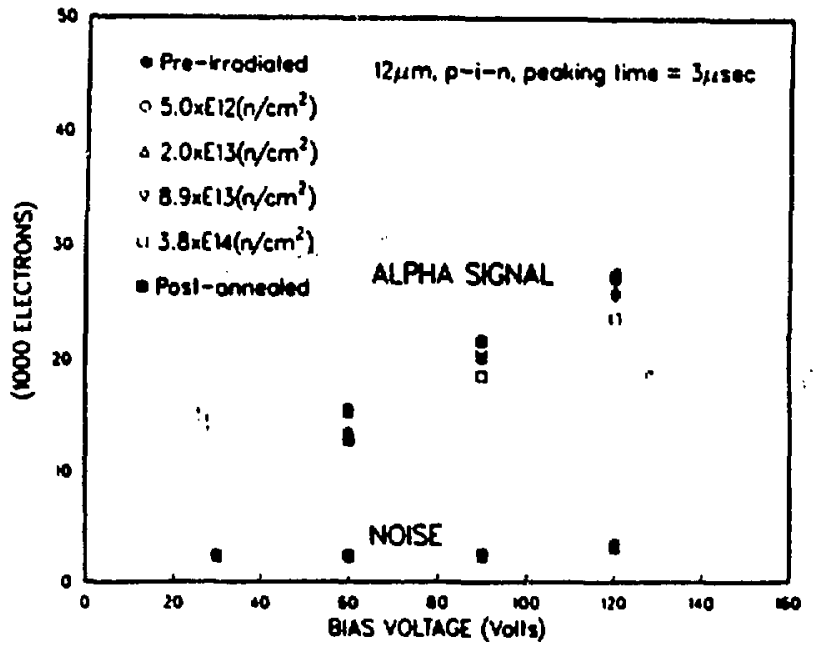




\section{THIN FILM TRANSISTOR READOUT ELECTRONICS}

The capability of making thin film distributed electronics amplifiers and of coupling them to the pixel detector arrays as shown in Fig. 1 provides very attractive prospects for this technology. At present we are engaged in making various measurements of a-Si:H and polysilicon T.F.T. in order to assess their usefulness for the detector field.[15] In Table I below we show estimates of the characteristics of various T.F.T. technologies. A major unknown has been the noise level that aSi:H or polysilicon input amplifier stage would contribute to the noise of a pixel detector. Our preliminary measurements, shown here, indicate that this noise -- while larger than that of xtal silicon MOSFETS is low enough to be acceptable.

\section{Table I}

\begin{tabular}{|c|c|c|c|c|c|c|}
\hline $\begin{array}{c}\text { Tyos of } \\
\text { TPT }\end{array}$ & 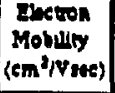 & WIL & $\underset{(\mu N \boldsymbol{m})}{8_{m}}$ & $\begin{array}{c}\text { Fnquncy } \\
\text { Lumt (3db) } \\
\text { (MHa) }\end{array}$ & $\begin{array}{c}\text { Nose * } \\
(1 \text { hoes) } \\
(\text { olestons })\end{array}$ & $\begin{array}{c}\text { Raduown } \\
\text { Restousnce }\end{array}$ \\
\hline $\begin{array}{c}\text { e-sith } \\
10 \mu \mathrm{m} \text { Tech }\end{array}$ & $0.3 \cdot 0.1$ & $2 \cdot 20$ & $2 \cdot 4$ & 1.5 & $\sim 350$ & Excellent \\
\hline $\begin{array}{c}-8141 \\
+\mu n \text { Tuch }\end{array}$ & $0.3 \cdot 0.8$ & $2-50$ & $2 \cdot 5$ & $3-10$ & $\sim 350$ & Excellont \\
\hline $\begin{array}{l}\text { a-still } \\
\text { Yarted }\end{array}$ & $0.3-0.0$ & $\begin{array}{l}100- \\
1000\end{array}$ & $30-300$ & $5=10$ & $\geq 1000$ & Unknown \\
\hline $\begin{array}{l}\text { Polyolitison } \\
\text { 400*Amand }\end{array}$ & $10-20$ & $2-20$ & $3 \cdot 30$ & $10-20$ & $\begin{array}{c}2500 \\
\text { (expected) }\end{array}$ & Unknawn \\
\hline $\begin{array}{l}\text { Pobsiticon } \\
\text { s00\%huned }\end{array}$ & $50-100$ & $2-20$ & $15=150$ & $50-100$ & 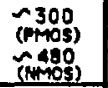 & Unknown \\
\hline $\begin{array}{c}\text { c-st od } \\
\text { Iaruinor } \\
\text { (301) }\end{array}$ & $\begin{array}{r}1000= \\
1500\end{array}$ & $2-20$ & $\begin{array}{l}1000- \\
2000\end{array}$ & $>100$ & $\begin{array}{l}\operatorname{Iam} 1 \\
\text {-SI RET }\end{array}$ & Excallont \\
\hline
\end{tabular}

- Noise values tre tiven for a typical size of each TFT with I usec CR-RC shapine amplifiers. However these measurements are preliminary because we have not measured enough samples.

The noise of a MOSFET in the frequency domain is given by the following formula[16].

$$
\overline{V_{i}^{3}}=4 K T \frac{2}{3} \frac{l}{8 m}+\frac{K}{C_{. f}}
$$

The first term is the Nyquist noise and the second is the $1 / f$ (flicker) noise. In the equations below, the detector noise sources, Nyquist, shor, $1 / f$ are combined together with the input stage amplifier noise and given as number of electrons $\mathrm{N}$ detected in a RC-CR shaping interval $\tau_{0}$ as given below [17].

$$
\overline{N_{\Delta}^{2}}=6.0 \times 10^{5} \frac{\left(C_{D}+C_{i}\right)^{2}}{\tau_{0} 8_{m}} \text { Nyquist noise }
$$

$C_{D}, C_{i}$, are the detector and FET input capacities in $\mathrm{pF}, \tau_{0}$ - shaping time constant in $\mu$ sec and $g_{m}=$ transconductance of the FET in $\mu \mathrm{A} / \mathrm{v}$.

The shot noise of the reverse bias current assumes the form

$$
\overline{N^{2}}=1.15 \times 10^{7} I \cdot \tau \text {, where } \mathrm{I} \text { is in } \mu \mathrm{A}
$$


The $1 / \mathrm{f}$ noise is given as

$$
\overline{N_{f}^{2}}=1.44 \times 10^{26} \frac{\left(C_{D}+C_{i}\right)^{2}}{C_{i}} \cdot K
$$

The $\Delta$ and $1 / f$ noise expressions show the need to keep the combined detector and FET input capacity low. Figure 12 shows measurements of the frequency dependence of noise from a-Si:H and from $2900^{\circ} \mathrm{C}$ anneal polysilicon TFT. In Table II below we give the expected noise numbers for a detector [ $300 \times 300 \mu \mathrm{m} \times 50 \mu \mathrm{m}$ thick] TFT combination.

The l/f noise from various types of TFT's is the largest noise component and indicates that continuing research to decrease this would be a useful avenue for continued development.

Table II

\begin{tabular}{|c|c|c|c|c|}
\hline \multicolumn{2}{|c|}{ Noise (e) } & \multirow{2}{*}{$\begin{array}{c}\text { a-Si:H TFT } \\
77 \\
72 \\
7 \\
24 \\
107 \\
340\end{array}$} & \multirow{2}{*}{ 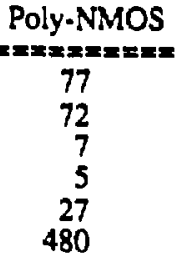 } & \multirow{2}{*}{$\begin{array}{c}\text { Poly-PMOS } \\
77 \\
72 \\
7 \\
5 \\
33 \\
280\end{array}$} \\
\hline $\begin{array}{l}v_{L D} \\
v_{S D} \\
V_{F D} \\
v_{L T} \\
V_{T T} \\
V_{F T}\end{array}$ & $\begin{array}{l}\text { (Thermal noise of } R_{L} \text { ) } \\
\text { (Shot noise of detector) } \\
\text { (1/f noise of detector) } \\
\text { (Thermal noise of RD) } \\
\text { (Thermal noise of TFT) } \\
\text { (1/f noise of TFT) }\end{array}$ & & & \\
\hline 10 & $\begin{array}{l}\text { m detector) } \\
\text { e) }\end{array}$ & $\begin{array}{r}3000 \\
370 \\
8.1\end{array}$ & 30 & $\begin{array}{r}3000 \\
300 \\
10 .\end{array}$ \\
\hline
\end{tabular}
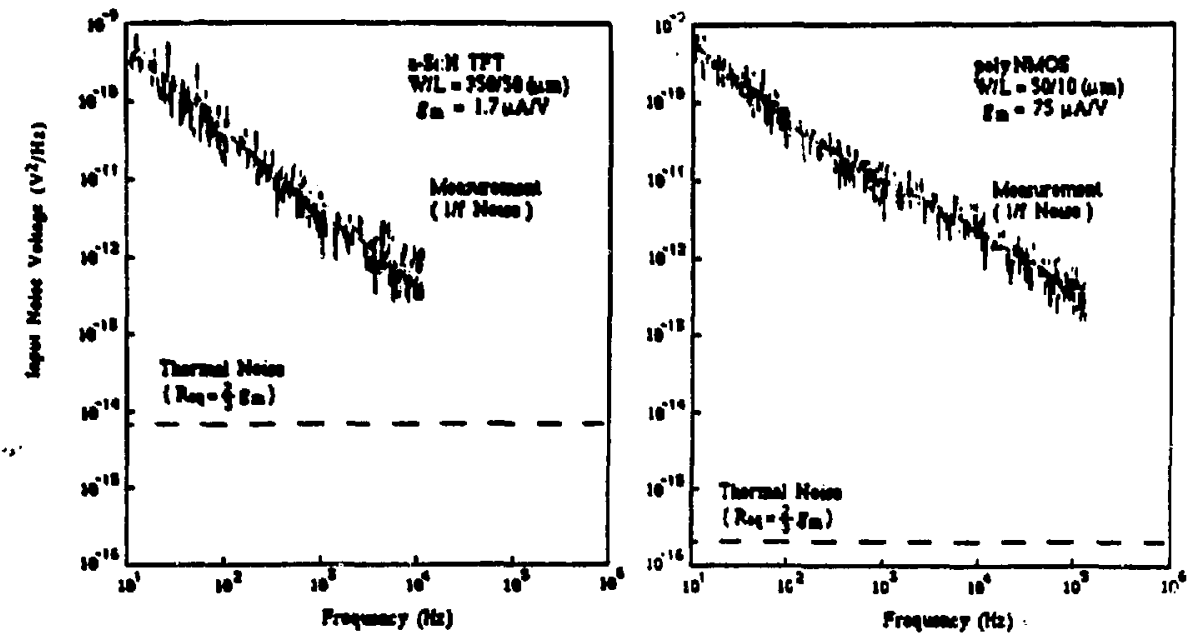

Fig. 12 Flicker (1/f) and Nyquist noise in a-Si:H and poly silicon T.F.T. 


\section{ACKNOWLEDGEMENTS}

We would like to express our appreciation to C.C. Tsai, M. Hack and A. Lewis of Xerox PARC. We also thank A. Madan, J. Xi and R. Hollingsworth of Glasstech-Solar, for making the p-i-n thick samples. We are also grateful to L. Lawler and F. Kirsten of LBL for electronics assistance. This work was supported by the Director, Office of Energy Research, Office of High Energy and Nuclear Physics, Division of High Energy Physics of the U.S. Department of Energy under contract \#DE-AC03-76SF00098.

\section{REFERENCES}

(1) V. Perez-Mendez, J. Morel, S.N. Kaplan, R.A. Street, NIM A252 (1986) 478.

(2) S. Qureshi, V. Perez-Mendez, S.N. Kaplan, I. Fujieda, G. Cho and R.A. Street, Paper E. 10.4 (LBL-26997) Presented at MRS Conference, San Diego (April 1989).

(3) S. Qureshi, V. Perez-Mendez, S.N. Kaplan, I. Fujieda, G. Cho and R.A. Street, IEEE Trans. Nuc. Sci. (1989) NS 36 (1989) 194.

(4) V. Ferez-Mendez, S.N. Kaplan, W. Ward, S. Qureshi, and R.A. Street, NIM A260 (1987) 195.

(5) B. Equer and A. Karar, NIM A271 (1988) 574 1988. J. Dubeau et al., MRS Proc. 118 (1988) 439.

(6) V. Perez-Mendez, S.N. Kaplan, O. Cho, I. Fujieda, S. Qureshi, W. Waro and R.A. Street. NIM A 273(1988) 127.

(7) S.N. Kaplan, I. Fujieda, V. Perez-Mendez, S. Qureshi, W. Ward and R.A. Street, NIM A273 (1988) 611.

(8) C.A. Klein, J. App. Phy. 39 (1968) 2029.

(9) J.XI , R.E. Hollingsworth and A. Madan, presented at Super Collider Conference New Orleans, LA. (Feb. 1989).

(10) J. Coleman, Photonics Spectra 21 \#6 (June 1987).

(11) 1. Holl, E. Lorenz, G. Mageras, IEEE Trans. Nuc. Sci. NS 35(1988) 105.

(12) A.L.N. Stevens and A.D.M. Schrama de Pauw Phillips Res. Repts 29 (1974)340, $29(1974) 353$.

(13) S. Kaneko, F. Okumura, M. Sakamoto, H. Uchida, Y. Kajiwara, SPIE Vol 167, Amorphous Semiconductors for Microelectronics (1986) p. 127.

(14) R.Schwarz, J. Kolodzey, S. Aljishi, S. Wagner, Proc. 18th IEEE PVSC (1985) 903.

(15) V. Perez-Mendez, G. Cho, I. Fujieda, S.N. Kaplan, S. Qureshi and R.A. Street, Lawrence Berkeley Laboratory, Report LBL-26254 (April 1989).

(16) P.R. Gray and R.G. Meyer, "Analog Integrated Circuits", p. 666 (John Wiley \& Sons, 1986).

(17) F. Goulding and D. Landis, IEEE Trans. Nuc. Sci, NS29(1982)1125. 